\title{
An Unconditionally Stable Method for Solving the Acoustic Wave Equation
}

\author{
Zhi-Kai Fu, Li-Hua Shi, Zheng-Yu Huang, and Shang-Chen Fu \\ National Key Laboratory on Electromagnetic Environmental Effects and Electro-optical Engineering, \\ PLA University of Science and Technology, Nanjing 210007, China
}

Correspondence should be addressed to Li-Hua Shi; shilhnj@163.com

Received 16 April 2015; Revised 21 July 2015; Accepted 26 July 2015

Academic Editor: Mitsuhiro Okayasu

Copyright (C) 2015 Zhi-Kai Fu et al. This is an open access article distributed under the Creative Commons Attribution License, which permits unrestricted use, distribution, and reproduction in any medium, provided the original work is properly cited.

An unconditionally stable method for solving the time-domain acoustic wave equation using Associated Hermit orthogonal functions is proposed. The second-order time derivatives in acoustic wave equation are expanded by these orthogonal basis functions. By applying Galerkin temporal testing procedure, the time variable can be eliminated from the calculations. The restriction of Courant-Friedrichs-Levy (CFL) condition in selecting time step for analyzing thin layer can be avoided. Numerical results show the accuracy and the efficiency of the proposed method.

\section{Introduction}

Numerical simulation of the acoustic wave equation has been widely used in many areas, such as geophysics, exploration, and ultrasonic detection [1-5]. The finite difference (FD) method in time domain is one of the most widely used methods in solving problems related to acoustics due to its efficiency and simplicity [6-8]. However, as the FD method is an explicit time-marching algorithm, the time step must be limited by the Courant-Friedrichs-Levy (CFL) condition, which means that the computation time will increase dramatically as the spatial grid becomes small. Hence, some unconditionally stable methods were proposed to solve this problem [9-12]. The main categories of these methods include the alternating-direction implicit (ADI) method [10] and the orthogonal decomposition method using weighted Laguerre polynomials (WLP) [11]. Recently, Huang et al. [12, 13] developed a new orthogonal decomposition method using Associated Hermit orthogonal functions and have applied it to first-order Maxwell's equation.

In this paper, we extended the unconditionally stable method using $\mathrm{AH}$ functions to solve the second-order acoustic wave equation. Firstly, the second-order time derivatives in the acoustic wave equation are expanded by a set of orthogonal basis functions. Secondly, the time variable can be eliminated from the calculations by using Galerkin's method.
Finally, a set of implicit equations in the whole computational domain is established and solved in the AH domain.

\section{Numerical Formulation for the Acoustic Wave Equation}

2.1. 1D Acoustic Wave Equation. For simplicity, we consider the $1 \mathrm{D}$ acoustic wave equation with an external source in isotropic media

$$
\begin{aligned}
\frac{\partial^{2} U}{\partial t^{2}} & =v^{2}\left(\frac{\partial^{2} U}{\partial x^{2}}\right)+f(x, t), \\
U(x, 0) & =0,0<x<l, 0<t<T, \\
\frac{\partial U(x, 0)}{\partial t} & =0,
\end{aligned}
$$

where $v$ is the sound velocity, $U$ is the wave displacement, and $f$ is an external source. In order to truncate the computational domain, we choose Mur's first-order absorbing boundary 
condition $(\mathrm{ABC})$ for infinite problem from [14]. At the boundary points $x_{0}=0$ or $x_{L}=l$, we have

$$
\left[\frac{\partial U}{\partial x}-\frac{1}{c} \frac{\partial U}{\partial t}\right]_{x=0 \text { or } l}=0 .
$$

Consider a set of modified $\mathrm{AH}$ orthogonal basis functions given by [12]

$$
\phi_{n}(t)=\frac{\left(2^{n} n ! \sigma \pi^{1 / 2}\right)^{-1 / 2} e^{-\bar{t}^{2} / 2}}{(-1)^{n} e^{\bar{t}^{2}}\left(d^{n} / d t^{n}\right)\left(e^{-\bar{t}^{2}}\right)},
$$

where $\bar{t}=\left(t-T_{f}\right) / \sigma$ is the transformed time variable, $T_{f}$ is the time-translating parameter, and $\sigma$ is the time-scaling parameter. By selecting proper $T_{f}$ and $\sigma$, the modified $\mathrm{AH}$ orthogonal basis functions can be used to approximate the causal displacement $U(x, t)$ by

$$
U(x, t)=\sum_{n=0}^{N-1} u^{n} \phi_{n}(t)
$$

where $u^{n}=\int_{-\infty}^{\infty} U(x, t) \varphi_{n}(t) d t$ is the $n$th expansion coefficient; $N$ is selected according to the bandwidth of the analyzed signal [15].

With the time derivation property of AH functions [15]

$$
\begin{aligned}
& \frac{d}{d t} \varphi_{n}(t) \\
& \quad= \begin{cases}-\frac{1}{\sigma} \sqrt{\frac{1}{2}} \varphi_{1}(t) & (n=0) \\
\frac{1}{\sigma} \sqrt{\frac{n}{2}} \varphi_{n-1}(t)-\frac{1}{\sigma} \sqrt{\frac{n+1}{2}} \varphi_{n+1}(t) & (n \geq 1) .\end{cases}
\end{aligned}
$$

We can express the first-order time derivation and the second-order time derivation of the displacement $U(x, t)$ as

$$
\begin{gathered}
\frac{\partial U(x, t)}{\partial t}=\sum_{n=0}^{\infty}\left(u^{n+1}(x) \sqrt{\frac{n+1}{2 \sigma^{2}}}-u^{n-1}(x) \sqrt{\frac{n}{2 \sigma^{2}}}\right) \\
\cdot \phi_{n}(t), \\
\frac{\partial^{2}}{\partial t^{2}} U(x, t)=\sum_{n=0}^{\infty}\left(u^{n+2}(x) \sqrt{\frac{n+2}{2 \sigma^{2}}} \sqrt{\frac{n+1}{2 \sigma^{2}}}\right. \\
\left.-\frac{2 n+1}{2 \sigma^{2}} u^{n}(x)+u^{n-2}(x) \sqrt{\frac{n-1}{2 \sigma^{2}}} \sqrt{\frac{n}{2 \sigma^{2}}}\right) \phi_{n}(t),
\end{gathered}
$$

where $u^{-2}(x)=0, u^{-1}(x)=0$, and $n$ represents the order in the AH domain. The deduction can be found in the Appendix.

Substituting (4) and (7) into (1), we obtain

$$
\begin{aligned}
& \sum_{n=0}^{\infty}\left(u^{n+2}(x) \sqrt{\frac{n+2}{2 \sigma^{2}}} \sqrt{\frac{n+1}{2 \sigma^{2}}}-\frac{2 n+1}{2 \sigma^{2}} u^{n}(x)\right. \\
& \left.+u^{n-2}(x) \sqrt{\frac{n-1}{2 \sigma^{2}}} \sqrt{\frac{n}{2 \sigma^{2}}}\right) \phi_{n}(t)=v^{2} \sum_{n=0}^{\infty} \frac{\partial^{2}}{\partial x^{2}} u^{n}(x) \\
& \cdot \phi_{n}(t)+\sum_{n=0}^{\infty} f^{n}(x) \phi_{n}(t) .
\end{aligned}
$$

Based on the orthogonal property of the $\mathrm{AH}$ functions, we can obtain equations of the AH expansion coefficients using the temporal Galerkin testing procedure [11]

$$
\begin{gathered}
u^{n+2}(x) \sqrt{\frac{n+2}{2}} \sqrt{\frac{n+1}{2}}-\frac{2 n+1}{2} u^{n}(x) \\
+u^{n-2}(x) \sqrt{\frac{n-1}{2}} \sqrt{\frac{n}{2}} \\
=v^{2} \sigma^{2} \frac{\partial^{2}}{\partial x^{2}} u^{n}(x)+\sigma^{2} f^{n}(x),
\end{gathered}
$$

where $f^{n}(x)=\int_{-T_{s} / 2}^{T_{s} / 2} f(x, t) \varphi_{n}(t) d t, n=0,1,2, \ldots$. We can partition the spatial domain $[0, l]$ into $0=x_{1}<x_{1}<\cdots<$ $x_{L}=l$. Then the spatial discrete form of (9) at point $x_{i}(0<$ $i<L$ ) can be written as

$$
\begin{gathered}
u_{i}^{n+2} \sqrt{\frac{n+2}{2}} \sqrt{\frac{n+1}{2}}-\frac{2 n+1}{2} u_{i}^{n}+u_{i}^{n-2} \sqrt{\frac{n-1}{2}} \sqrt{\frac{n}{2}} \\
=\frac{v^{2} \sigma^{2}}{\Delta x^{2}}\left(u_{i+1}^{n}-2 u_{i}^{n}+u_{i-1}^{n}\right)+\sigma^{2} f_{i}^{n},
\end{gathered}
$$

where $\Delta x$ is the spatial grid size.

In (10), a set of implicit equations is included in the $\mathrm{AH}$ domain. Based on the recurrence relation from the $n-2$ th order coefficients and the $n$th order coefficients to the $n+2$ th order coefficients, we can rewrite (10) in a matrix form:

$$
\begin{aligned}
& -\frac{v^{2} \sigma^{2}}{\Delta x^{2}}[\beta][U]_{i+1}+[\alpha][U]_{i}-\frac{v^{2} \sigma^{2}}{\Delta x^{2}}[\beta][U]_{i-1} \\
& \quad=\sigma^{2}[\beta][f]_{i}
\end{aligned}
$$

where $[\beta]=I_{N \times N}$ in a unit matrix. Consider

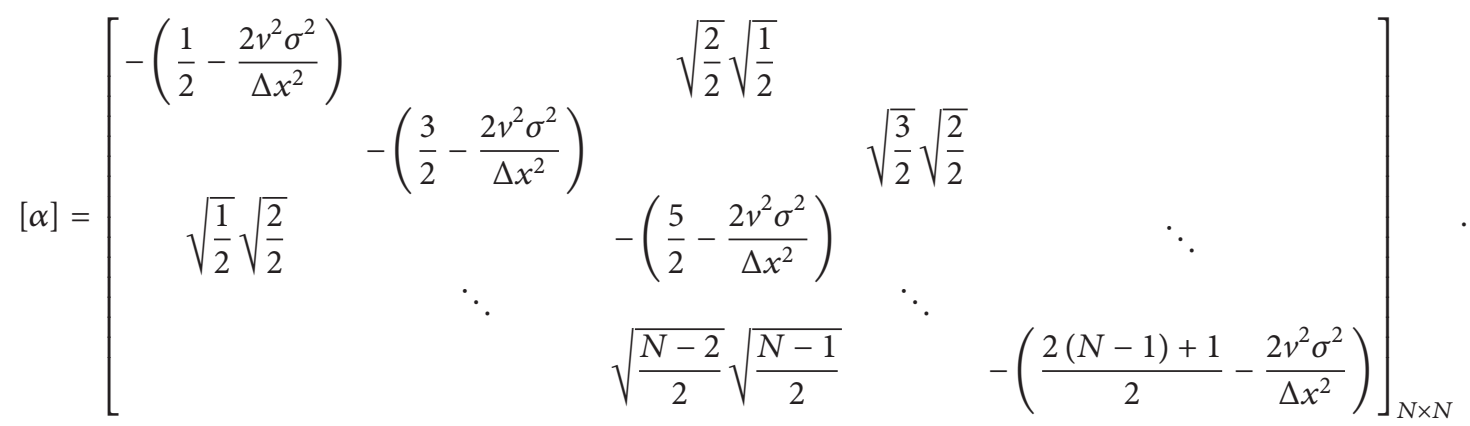


Here, $[\alpha]$ is a tridiagonal coefficient matrix. $[U]_{i}=$ $\left(u_{i}^{0}, u_{i}^{1}, \ldots, u_{i}^{N-1}\right)^{T}$ and $[f]_{i}=\left(f_{i}^{0}, f_{i}^{1}, \ldots, f_{i}^{N-1}\right)^{T}$ are the $N$ tuple representations in AH subspace for the displacement and the external source, respectively. In (11), we can find each point has a relationship with its adjacent two points, and a set of implicit equations can be obtained in the whole computation domain from point $x_{1}$ to $x_{L}$ :

$$
[A][U]=[J],
$$

where

$$
=\left[\begin{array}{ccccc}
{[\alpha]} & -\frac{v^{2} \sigma^{2}}{\Delta x^{2}}[\beta] & & & \\
-\frac{v^{2} \sigma^{2}}{\Delta x^{2}}[\beta] & {[\alpha]} & -\frac{v^{2} \sigma^{2}}{\Delta x^{2}}[\beta] & & \\
& -\frac{v^{2} \sigma^{2}}{\Delta x^{2}}[\beta] & {[\alpha]} & -\frac{v^{2} \sigma^{2}}{\Delta x^{2}}[\beta] & \\
& & \ddots & \ddots & \ddots \\
& & & -\frac{v^{2} \sigma^{2}}{\Delta x^{2}}[\beta] & {[\alpha]}
\end{array}\right]_{L \times L}
$$

is a tridiagonal matrix with submatrix elements. $[U]$ is a combination of all $[U]_{i}$ in the whole computation domain. $[J]$ is a vector related with external source expansion coefficients for all points.

Substitute (4) and (6) into (2) and eliminate the temporal terms to obtain (at $x=x_{L}$ )

$$
\begin{aligned}
& \frac{\partial}{\partial x} U^{n}(x)-\frac{1}{c}\left(u^{n+1}(x) \sqrt{\frac{n+1}{2 \sigma^{2}}}-u^{n-1}(x) \sqrt{\frac{n}{2 \sigma^{2}}}\right) \\
& \quad=0 .
\end{aligned}
$$

Using the average techniques, we have

$$
\begin{aligned}
U^{n}\left(L-\frac{1}{2}\right) & =\frac{U^{n}(L-1)+U^{n}(L)}{2}, \\
\frac{\partial}{\partial x} U^{n}\left(L-\frac{1}{2}\right) & =\frac{U^{n}(L)-U^{n}(L-1)}{\Delta x} .
\end{aligned}
$$

Substituting (16) into (15), we have

$$
\begin{aligned}
& \frac{2 c \sigma}{\Delta x} U_{L}^{n}-\sqrt{\frac{n+1}{2}} U_{L}^{n+1}+\sqrt{\frac{n}{2}} U_{L}^{n-1} \\
& \quad=\frac{2 c \sigma}{\Delta x} U_{L-1}^{n}+\sqrt{\frac{n+1}{2}} U_{L-1}^{n+1}-\sqrt{\frac{n}{2}} U_{L-1}^{n-1} .
\end{aligned}
$$
obtain

Applying (17) into all orders in the AH domain, we can

$$
\left(\frac{2 v \sigma}{\Delta x}[\beta]+[\varepsilon]\right)[U]_{L}-\left(\frac{2 v \sigma}{\Delta x}[\beta]-[\varepsilon]\right)[U]_{L-1}=0,
$$

where

$$
[\varepsilon]=\left[\begin{array}{cccc}
\sqrt{\frac{1}{2}}^{-\sqrt{\frac{1}{2}}} & & \\
& \ddots & & \\
& & \sqrt{\frac{N-1}{2}}^{-\sqrt{\frac{N-1}{2}}}
\end{array}\right]_{N \times N} .
$$

Using the technique above, the absorbing boundary condition at point $x=x_{1}$ can be expressed as

$$
\left(\frac{2 v \sigma}{\Delta x}[\beta]+[\varepsilon]\right)[U]_{1}-\left(\frac{2 v \sigma}{\Delta x}[\beta]-[\varepsilon]\right)[U]_{2}=0 .
$$

Introducing the boundary condition (18) and (20) into (11), (13) can be replaced by

$$
[\bar{A}][U]=[\bar{J}]
$$

where $[\bar{A}]$ is an adaptive coefficient matrix including absorbing boundary condition and $[\bar{J}]$ is a coefficient matrix corresponding to $[\bar{A}]$.

We can use the lower-upper (LU) decomposition method to decompose $[\bar{A}]$ and use the back-substitution method to calculate the coefficients of the displacement for all points. The displacement $U(x, t)$ can be reconstructed finally by using (4).

2.2. 2D Acoustic Wave Equation. Considering the need for practical application, we extend the method to the $2 \mathrm{D}$ acoustic wave equation, which is given by

$$
\begin{gathered}
\frac{\partial^{2} U}{\partial t^{2}}=v^{2}\left(\frac{\partial^{2} U}{\partial x^{2}}+\frac{\partial^{2} U}{\partial y^{2}}\right)+f(x, y, t), \\
\quad(x, y) \in \Omega \times[0, T], \\
U(x, y, 0)=0, \quad \frac{\partial U(x, y, 0)}{\partial t}=0,
\end{gathered}
$$

where $\Omega=\left[0, l_{1}\right] \times\left[0, l_{2}\right]$ is divided into $N_{x} \times N_{y}$ grid.

Substituting (4) and (7) into (22) and rearranging it, we can obtain a matrix form equation which is similar to (11):

$$
\begin{aligned}
- & \frac{v^{2} \sigma^{2}}{\Delta x^{2}}[\beta][U]_{i-1, j}-\frac{v^{2} \sigma^{2}}{\Delta x^{2}}[\beta][U]_{i+1, j}+[\alpha][U]_{i, j} \\
& -\frac{v^{2} \sigma^{2}}{\Delta y^{2}}[\beta][U]_{i, j-1}-\frac{v^{2} \sigma^{2}}{\Delta y^{2}}[\beta][U]_{i, j-1} \\
= & v^{2} \sigma^{2}[\beta][f]_{i, j},
\end{aligned}
$$

where $\Delta x$ is the spatial grid size in $x$ direction and $\Delta y$ is the spatial grid size in $y$ direction. In (23), we can find each point has a relationship with its adjacent four points, so matrix $[A]$ is a five-diagonal coefficient matrix in $2 \mathrm{D}$ case.

For the absorbing boundary condition, the boundary condition can be obtained by using (15) to (20). But the corner-point needs special treatment and the spatial grid size is adjusted to $h=\sqrt{\Delta x^{2}+\Delta y^{2}}$ at corner-point. For the four 


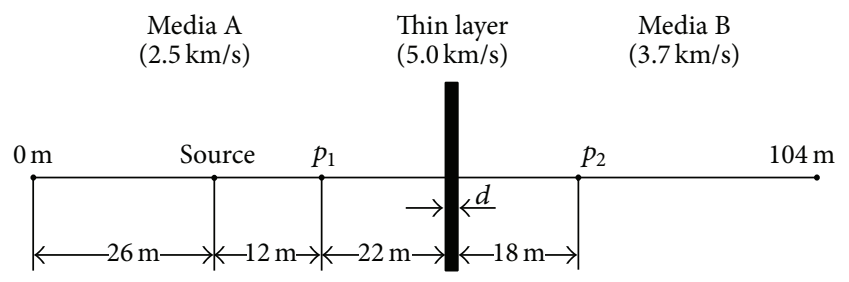

Figure 1: Configuration of a thin layer $(d=0.1 \mathrm{~m})$ embedded in two isotropic media.

corner-points, Mur's absorbing boundary condition can be expressed as

$$
\begin{aligned}
& \left(\frac{2 v \sigma}{h}[\beta]+[\varepsilon]\right)[U]_{1.1}-\left(\frac{2 v \sigma}{h}[\beta]-[\varepsilon]\right)[U]_{2.2}=0 \\
& \left(\frac{2 v \sigma}{h}[\beta]+[\varepsilon]\right)[U]_{1, N_{y}} \\
& \quad-\left(\frac{2 v \sigma}{h}[\beta]-[\varepsilon]\right)[U]_{2, N_{y}-1}=0 \\
& \left(\frac{2 v \sigma}{h}[\beta]+[\varepsilon]\right)[U]_{N_{x}, 1} \\
& \quad-\left(\frac{2 v \sigma}{h}[\beta]-[\varepsilon]\right)[U]_{N_{x}-1,2}=0 \\
& \left(\frac{2 v \sigma}{h}[\beta]+[\varepsilon]\right)[U]_{N_{x}, N_{y}} \\
& -\left(\frac{2 v \sigma}{h}[\beta]-[\varepsilon]\right)[U]_{N_{x}-1, N_{y}-1}=0
\end{aligned}
$$

Introducing Mur's absorbing boundary condition, the coefficient matrix $[A]$ can be adjusted to $[\bar{A}]$. Using the lowerupper (LU) decomposition method and the back-substitution method, the coefficients of all points can be calculated. And the displacement $U(x, y, t)$ can be reconstructed finally using the method described in $1 \mathrm{D}$ case.

\section{Numerical Results}

3.1. 1D Case. In order to validate the proposed method, a 1D structure constituted by two isotropic media separated by a thin layer $(d=0.1 \mathrm{~m}, v=5.0 \mathrm{~km} / \mathrm{s})$ is analyzed. As shown in Figure 1, the thin layer is located at $60 \mathrm{~m}$, and the total simulation length is $104 \mathrm{~m}$. Displacement $U(x, t)$ at two points $p_{1}(38 \mathrm{~m})$ and $p_{2}(78.1 \mathrm{~m})$ is observed. The sound velocity in media $A$ is $2.5 \mathrm{~km} / \mathrm{s}$, and the sound velocity in media B is $3.7 \mathrm{~km} / \mathrm{s}$. Nonuniform spatial grids are used. In the thin layer $\Delta x=0.01 \mathrm{~m}$; in other parts $\Delta x=1 \mathrm{~m}$.

The external source is located at $x=26 \mathrm{~m}$ and its expression is $f(t)=-5.76 f_{0}^{2}\left[1-16\left(0.6 f_{0} t-1\right)^{2}\right] \exp \left[-8\left(0.6 f_{0} t-\right.\right.$ $1)^{2}$ ], where $f_{0}=100 \mathrm{~Hz}$. Time support for the simulation is $0.16 \mathrm{~s}$. To expand $f(t)$ in AH domain, we select $N=52$, $\sigma=0.008$, and $T_{f}=0.5$. Due to the limitation of the

\begin{tabular}{|c|c|c|}
\hline & \multicolumn{2}{|c|}{$\Delta x=0.01 \mathrm{~m}$} \\
\hline & $\Delta t$ & CPU time \\
\hline FD method & $2 \times 10^{-6} \mathrm{~s}$ & $3.198 \mathrm{~s}$ \\
\hline Proposed method & $2 \times 10^{-3} \mathrm{~s}$ & $0.3127 \mathrm{~s}$ \\
\hline
\end{tabular}
CFL condition, the time step is set as $2 \times 10^{-6} \mathrm{~s}$ for the FD method under the condition of $\Delta x=0.01 \mathrm{~m}$. Time step is not involved in $\mathrm{AH}$ domain computation. However, for $\mathrm{AH}$ decomposition, we still need to specify a sample interval; the time step $\Delta t=0.002 \mathrm{~s}$ is good enough. The absolute error
TABLE 1: The comparison of CPU time.

TABLE 2: The comparison of CPU time.

\begin{tabular}{lcc}
\hline & \multicolumn{2}{c}{$\Delta x=0.001 \mathrm{~m}$} \\
& $\Delta t$ & CPU time \\
\hline FD method & $1.33 \times 10^{-7} \mathrm{~s}$ & $237.9171 \mathrm{~s}$ \\
Proposed method & $5 \times 10^{-4} \mathrm{~s}$ & $30.8813 \mathrm{~s}$ \\
\hline
\end{tabular}

$R$ is defined as $R=\left|U^{a}(t)-U^{c}(t)\right|$ for comparison of the two methods. $U^{c}(t)$ is the reference data obtained from the FD method with $\Delta x=0.01 \mathrm{~m}$, and $U^{a}(t)$ is the numerical result obtained from the FD method $(\Delta x=0.1 \mathrm{~m})$ and the proposed method $(\Delta x=0.01 \mathrm{~m})$. The numerical results at $p_{1}$ and their errors are shown in Figure 2(a). Figure 2(b) is the numerical results at $p_{2}$. From Figure 2, it can be seen that the proposed method has a good agreement with the FD method. The absolute error of the proposed method $(\Delta x=0.01 \mathrm{~m})$ is much lower than that of the FD method $(\Delta x=0.1 \mathrm{~m})$ at both $p_{1}$ and $p_{2}$.

Table 1 shows the comparison of CPU time between the proposed method and the FD method. Under the condition of $\Delta x=0.01 \mathrm{~m}$, the time step of the proposed method is 1000 times as large as that of the FD method, and the total CPU time for the proposed method can be reduced to about $10 \%$ of the FD method.

3.2. $2 \mathrm{D}$ Case. In this section, we show results from a $2 \mathrm{D}$ numerical modeling by the FD method and the proposed method. The 2D model is established by two isotropic media separated by a thin layer $(d=0.01 \mathrm{~m}, v=6.0 \mathrm{~km} / \mathrm{s})$, which is similar to the $1 \mathrm{D}$ case. As shown in Figure 3, the computational domain is $60 \mathrm{~m} \times 90 \mathrm{~m}$, and the thin layer is located at $72 \mathrm{~m}$ in $x$ direction. Displacement $U(x, y, t)$ at two points $p_{1}(36 \mathrm{~m}, 30 \mathrm{~m})$ and $p_{2}(81.01 \mathrm{~m}, 30 \mathrm{~m})$ is observed. The sound velocity in media $A$ is $3 \mathrm{~km} / \mathrm{s}$, and the sound velocity in media B is $4.7 \mathrm{~km} / \mathrm{s}$. Nonuniform spatial grids are used. In $x$ direction, $\Delta x=0.001 \mathrm{~m}$ in the thin layer, and $\Delta x=3 \mathrm{~m}$ in other parts. In $y$ direction, $\Delta y=3 \mathrm{~m}$. The external source is the same as the $1 \mathrm{D}$ case and is located at $s(27 \mathrm{~m}, 30 \mathrm{~m})$.

Time support for the simulation is $0.06 \mathrm{~s}$. The time step is set as $1.33 \times 10^{-7} \mathrm{~s}$ and $5 \times 10^{-4} \mathrm{~s}$ for the FD method and the proposed method, respectively. Figure 4 shows the $2 \mathrm{D}$ modeling records at $p_{1}$ and $p_{2}$ for the multilayer isotropic media model. The waveform corresponding to the proposed method is in good agreement with that by the FD method, which demonstrates that the proposed method has good accuracy. Table 2 shows the comparison of CPU time between the proposed method and the FD method. Under the condition of $\Delta x=0.001 \mathrm{~m}$, the time step of the proposed method is about 3700 times as large as that of the FD method, 

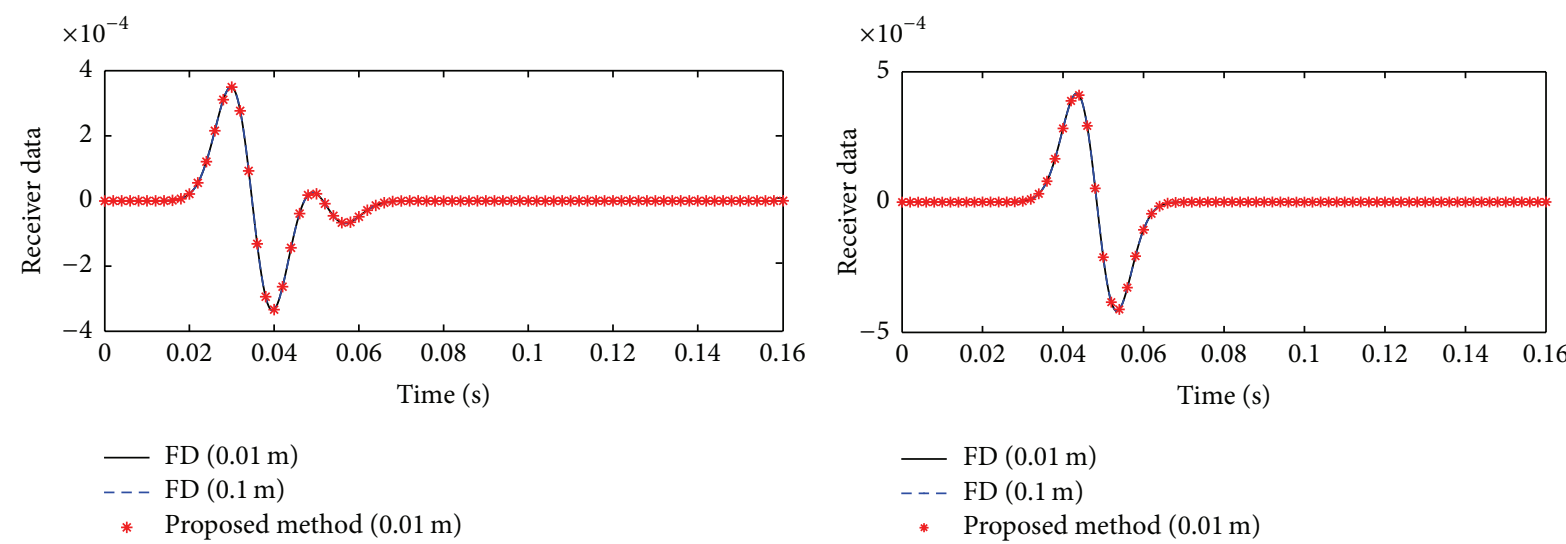

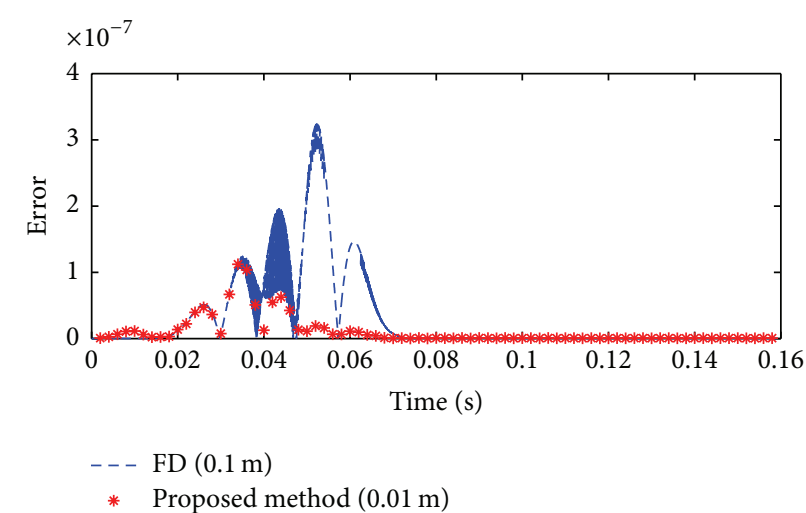

(a)

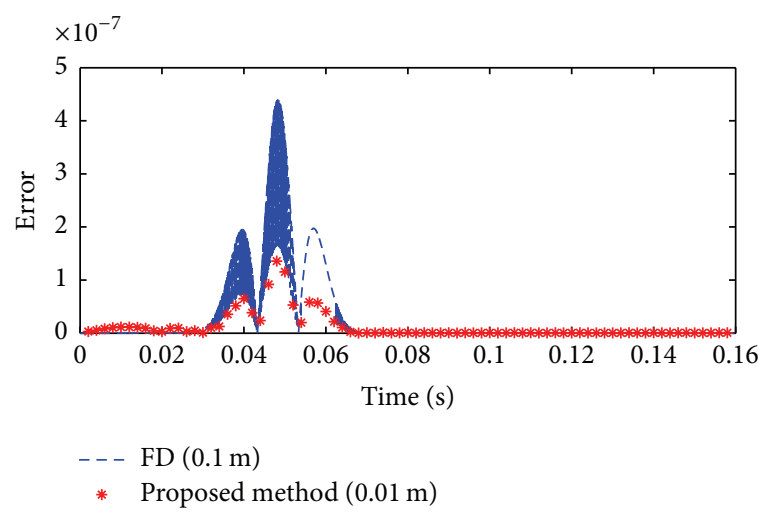

(b)

FIgURE 2: The numerical results and their errors at (a) $p_{1}$ and (b) $p_{2}$.

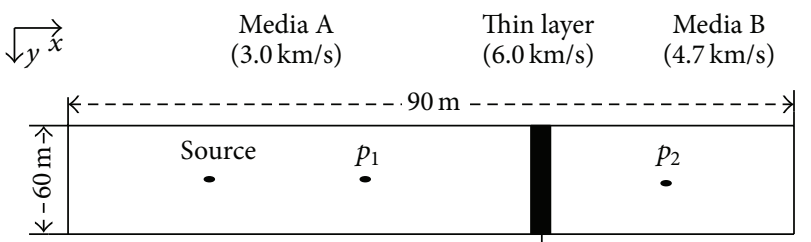

Fine structure $(d=0.01 \mathrm{~m})$

Figure 3: Configuration of a thin layer $(d=0.01 \mathrm{~m})$ embedded in two isotropic media.

and the total CPU time for the proposed method can be reduced to about $13 \%$ of the FD method.

\section{Conclusion}

In this paper, an unconditionally stable method has been proposed for solving the $1 \mathrm{D}$ and $2 \mathrm{D}$ acoustic wave equation in time domain. By applying Associated Hermit orthogonal function expansion and Galerkin temporal testing procedure, the time variable can be eliminated from the calculations and can make the proposed method unconditionally stable. The numerical results show the proposed method is efficient and the accuracy is still guaranteed.

\section{Appendix}

In order to deduce the second-order derivation, we deduce the first-order time derivation of the displacement first. Substituting (5) into the first-order time derivation of (4), we obtain

$$
\begin{gathered}
\frac{\partial}{\partial t} U(x, t)=\sum_{n=0}^{\infty} u^{n}(x) \frac{d}{d t} \phi_{n}(t)=u^{0}(x) \frac{d}{d t} \phi_{0}(t) \\
+\sum_{n=1}^{\infty} u^{n}(x) \frac{d}{d t} \phi_{n}(t)=u^{0}(x)\left(-\sqrt{\frac{1}{2 \sigma^{2}}} \phi_{1}(t)\right) \\
+\sum_{n=1}^{\infty} u^{n}(x)\left(\sqrt{\frac{n}{2 \sigma^{2}}} \phi_{n-1}(t)-\sqrt{\frac{n+1}{2 \sigma^{2}}} \phi_{n+1}(t)\right) \\
\stackrel{m=n-1}{=} u^{0}(x)\left(-\sqrt{\frac{1}{2 \sigma^{2}}} \phi_{1}(t)\right)+\sum_{m=0}^{\infty} u^{m+1}(x) \\
\cdot\left(\sqrt{\frac{m+1}{2 \sigma^{2}}} \phi_{m}(t)-\sqrt{\frac{m+2}{2 \sigma^{2}}} \phi_{m+2}(t)\right),
\end{gathered}
$$




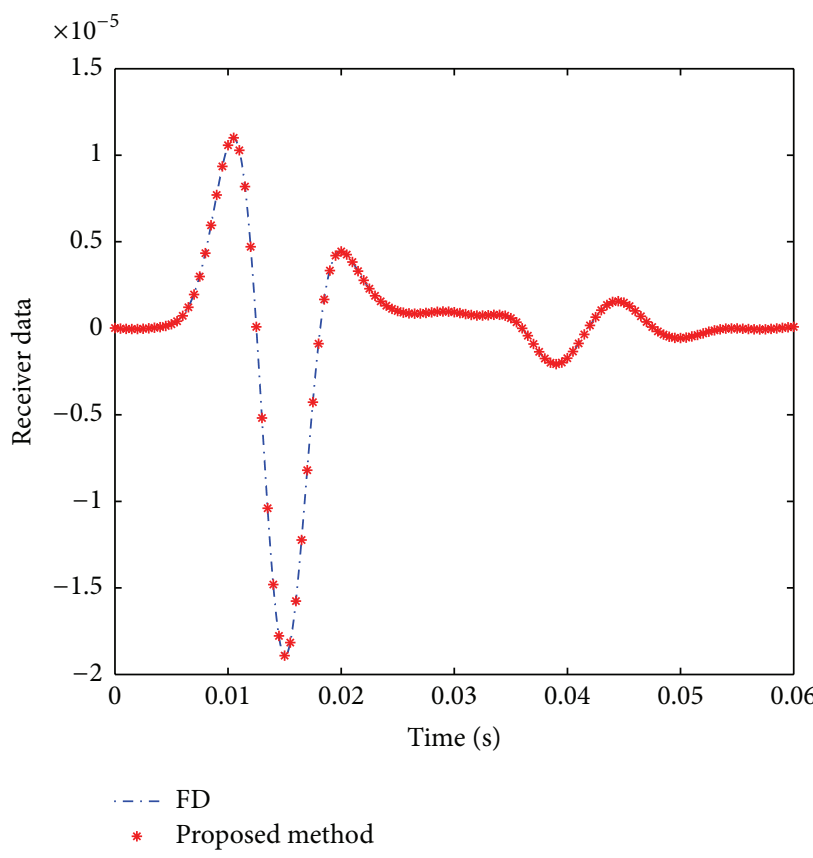

(a)

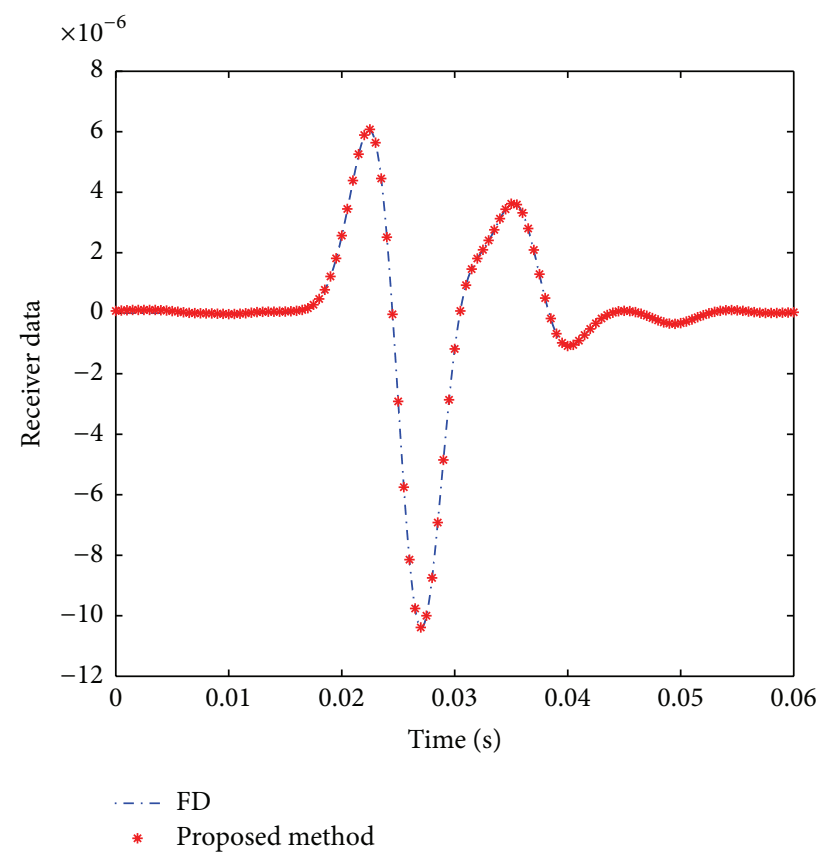

(b)

Figure 4: The numerical results at (a) $p_{1}$ and (b) $p_{2}$.

where

$$
\begin{aligned}
& u^{0}(x)\left(-\sqrt{\frac{1}{2 \sigma^{2}}} \varphi_{1}(t)\right) \\
& +\sum_{m=0}^{\infty} u^{m+1}(x)\left(-\sqrt{\frac{m+2}{2 \sigma^{2}}} \varphi_{m+2}(t)\right) \\
& \quad \stackrel{k=m+2}{=} u^{0}(x)\left(-\sqrt{\frac{1}{2 \sigma^{2}}} \varphi_{1}(t)\right) \\
& +\sum_{k=2}^{\infty} u^{k-1}(x)\left(-\sqrt{\frac{k}{2 \sigma^{2}}} \varphi_{k}(t)\right) \\
& =\sum_{k=0}^{\infty} u^{k-1}(x)\left(-\sqrt{\frac{k}{2 \sigma^{2}}} \varphi_{k}(t)\right)
\end{aligned}
$$

Then we have

$$
\begin{aligned}
& \frac{\partial}{\partial t} U(r, t)=\sum_{m=0}^{\infty} u^{m+1}(x)\left(\sqrt{\frac{m+1}{2 \sigma^{2}}} \varphi_{m}(t)\right) \\
& +\sum_{k=0}^{\infty} u^{k-1}(x)\left(-\sqrt{\frac{k}{2 \sigma^{2}}} \varphi_{k}(t)\right) \\
& \stackrel{m=n, k=n}{=} \sum_{n=0}^{\infty}\left(u^{n+1}(x) \sqrt{\frac{n+1}{2 \sigma^{2}}}-u^{n-1}(x) \sqrt{\frac{n}{2 \sigma^{2}}}\right) \\
& \cdot \varphi_{n}(t) .
\end{aligned}
$$

According to the method above, the second-order time derivation can be expressed as

$$
\begin{aligned}
& \frac{\partial^{2}}{\partial t^{2}} U(r, t) \\
& =\sum_{n=0}^{\infty}\left(u^{n+1}(x) \sqrt{\frac{n+1}{2 \sigma^{2}}}-u^{n-1}(x) \sqrt{\frac{n}{2 \sigma^{2}}}\right) \\
& \quad \cdot \frac{d}{d t} \varphi_{n}(t) \stackrel{m=n-1}{=} u^{1}(x) \sqrt{\frac{1}{2 \sigma^{2}}}\left(-\sqrt{\frac{1}{2 \sigma^{2}}} \varphi_{1}(t)\right) \\
& +\sum_{m=0}^{\infty}\left(u^{m+2}(x) \sqrt{\frac{m+2}{2 \sigma^{2}}}-u^{m}(x) \sqrt{\frac{m+1}{2 \sigma^{2}}}\right) \\
& \quad \cdot\left(\sqrt{\frac{m+1}{2 \sigma^{2}}} \varphi_{m}(t)-\sqrt{\frac{m+2}{2 \sigma^{2}}} \varphi_{m+2}(t)\right),
\end{aligned}
$$

where

$$
\begin{aligned}
& u^{1}(x) \sqrt{\frac{1}{2 \sigma^{2}}}\left(-\sqrt{\frac{1}{2 \sigma^{2}}} \varphi_{1}(t)\right) \\
& +\sum_{m=0}^{\infty}\left(u^{m+2}(x) \sqrt{\frac{m+2}{2 \sigma^{2}}}-u^{m}(x) \sqrt{\frac{m+1}{2 \sigma^{2}}}\right) \\
& \cdot\left(-\sqrt{\frac{m+2}{2 \sigma^{2}}} \varphi_{m+2}(t)\right) \stackrel{k=m+2}{=} u^{1}(x) \\
& \left.\cdot \sqrt{\frac{1}{2 \sigma^{2}}}\left(-\sqrt{\frac{1}{2 \sigma^{2}}} \varphi_{1}(t)\right) \stackrel{\sqrt{\frac{k-1}{2 \sigma^{2}}}}{ }\right) \\
& +\sum_{k=2}^{\infty}\left(u^{k}(x) \sqrt{\frac{k}{2 \sigma^{2}}}-u^{k-2}(x) \sqrt{ }\right.
\end{aligned}
$$




$$
\begin{aligned}
& \left(-\sqrt{\frac{k}{2 \sigma^{2}}} \varphi_{k}(t)\right) \\
& =\sum_{k=0}^{\infty}\left(u^{k}(x) \sqrt{\frac{k}{2 \sigma^{2}}}-u^{k-2}(x) \sqrt{\frac{k-1}{2 \sigma^{2}}}\right) \\
& \cdot\left(-\sqrt{\frac{k}{2 \sigma^{2}}} \varphi_{k}(t)\right) \\
& \left(\text { Let } u^{-2}(x)=0, u^{-1}(x)=0\right) .
\end{aligned}
$$

Then we have

$$
\begin{gathered}
\frac{\partial^{2}}{\partial t^{2}} U(x, t)=\sum_{m=0}^{\infty}\left(u^{m+2}(x) \sqrt{\frac{m+2}{2 \sigma^{2}}}\right. \\
\left.-u^{m}(x) \sqrt{\frac{m+1}{2 \sigma^{2}}}\right)\left(\sqrt{\frac{m+1}{2 \sigma^{2}}} \phi_{m}(t)\right) \\
+\sum_{k=0}^{\infty}\left(u^{k}(x) \sqrt{\frac{k}{2 \sigma^{2}}}-u^{k-2}(x) \sqrt{\frac{k-1}{2 \sigma^{2}}}\right) \\
\cdot\left(-\sqrt{\frac{k}{2 \sigma^{2}}} \phi_{k}(t)\right) \\
\quad+m=n, k=n \sum_{n=0}^{\infty}\left(u^{n+2}(x) \sqrt{\frac{n+2}{2 \sigma^{2}}} \sqrt{\frac{n+1}{2 \sigma^{2}}}\right. \\
\left.-\frac{2 n+1}{2 \sigma^{2}} u^{n}(x)+u^{n-2}(x) \sqrt{\frac{n-1}{2 \sigma^{2}}} \sqrt{\frac{n}{2 \sigma^{2}}}\right) \phi_{n}(t) .
\end{gathered}
$$

\section{Conflict of Interests}

The authors declare that there is no conflict of interests regarding the publication of this paper.

\section{References}

[1] D. D. Kosloff and E. Baysal, "Forward modeling by a Fourier method," Geophysics, vol. 56, pp. 231-241, 1982.

[2] D. H. Yang, P. Tong, and X. Y. Deng, "A central difference method with low numerical dispersion for solving the scalar wave equation," Geophysical Prospecting, vol. 60, no. 5, pp. 885905, 2012.

[3] R. M. Alford, K. R. Kelly, and D. M. Boore, "Accuracy of finitedifference modeling of the acoustic wave equation," Geophysics, vol. 39, no. 6, pp. 834-841, 1974.

[4] M. A. Dablain, "The application of high-order differencing to the scalar wave equation," Geophysics, vol. 51, no. 1, pp. 54-66, 1986.

[5] A. Ganguli, C. M. Rappaport, D. Abramo, and S. Wadia-Fascetti, "Synthetic aperture imaging for flaw detection in a concrete medium," NDT \& E International, vol. 45, no. 1, pp. 79-90, 2012.

[6] Y. Liu and M. K. Sen, "A new time-space domain highorder finite-difference method for the acoustic wave equation," Journal of Computational Physics, vol. 228, no. 23, pp. 87798806, 2009.
[7] S. Das, W. Liao, and A. Gupta, "An efficient fourth-order low dispersive finite difference scheme for a 2-D acoustic wave equation," Journal of Computational and Applied Mathematics, vol. 258, pp. 151-167, 2014.

[8] W. Y. Liao, "On the dispersion, stability and accuracy of a compact higher-order finite difference scheme for 3D acoustic wave equation," Journal of Computational and Applied Mathematics, vol. 270, pp. 571-583, 2014.

[9] R. K. Mohanty, "An unconditionally stable difference scheme for the one-space-dimensional linear hyperbolic equation," Applied Mathematics Letters, vol. 17, no. 1, pp. 101-105, 2004.

[10] R. K. Mohanty and M. K. Jain, "An unconditionally stable alternating direction implicit scheme for the two space dimensional linear hyperbolic equation," Numerical Methods for Partial Differential Equations, vol. 17, no. 6, pp. 684-688, 2001.

[11] Y.-S. Chung, T. K. Sarkar, B. H. Jung, and M. Salazar-Palma, "An unconditionally stable scheme for the finite-difference timedomain method," IEEE Transactions on Microwave Theory and Techniques, vol. 51, no. 3, pp. 697-704, 2003.

[12] Z.-Y. Huang, L.-H. Shi, B. Chen, and Y.-H. Zhou, "A new unconditionally stable scheme for FDTD method using associated Hermite orthogonal functions," IEEE Transactions on Antennas and Propagation, vol. 62, no. 9, pp. 4804-4809, 2014.

[13] H. Zheng-Yu, S. Li-Hua, Z. Ying-Hui, and C. Bin, "AnImproved paralleling-in-order solving scheme for AH-FDTD method using eigenvalue transformation," IEEE Transactions on Antennas and Propagation, vol. 63, no. 5, pp. 2135-2140, 2015.

[14] Z. Bi, K. Wu, C. Wu, and J. Litva, "A dispersive boundary condition for microstrip component analysis using the FDTD method," IEEE Transactions on Microwave Theory and Techniques, vol. 40, no. 4, pp. 774-777, 1992.

[15] L. R. Lo Conte, R. Merletti, and G. V. Sandri, "Hermite expansions of compact support waveforms: applications to myoelectric signals," IEEE Transactions on Biomedical Engineering, vol. 41, no. 12, pp. 1147-1159, 1994. 


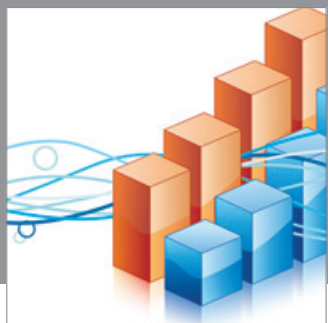

Advances in

Operations Research

mansans

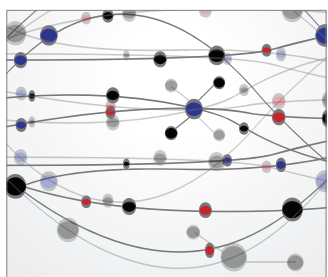

The Scientific World Journal
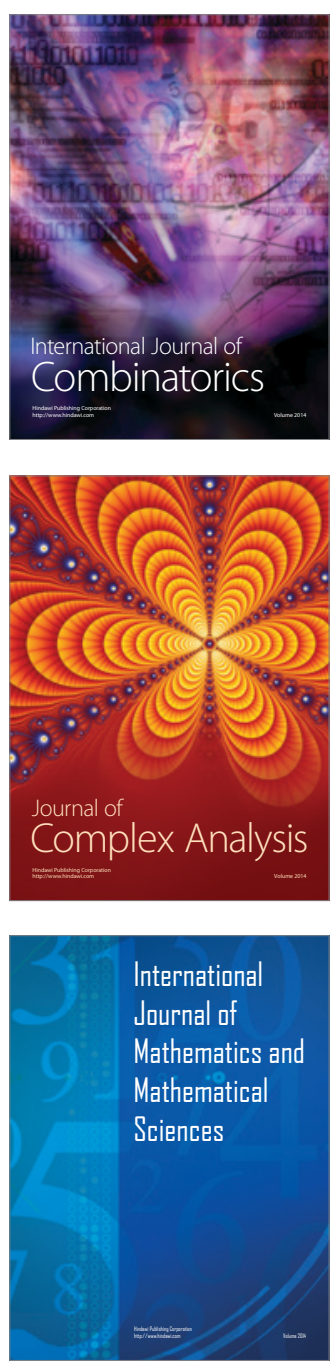
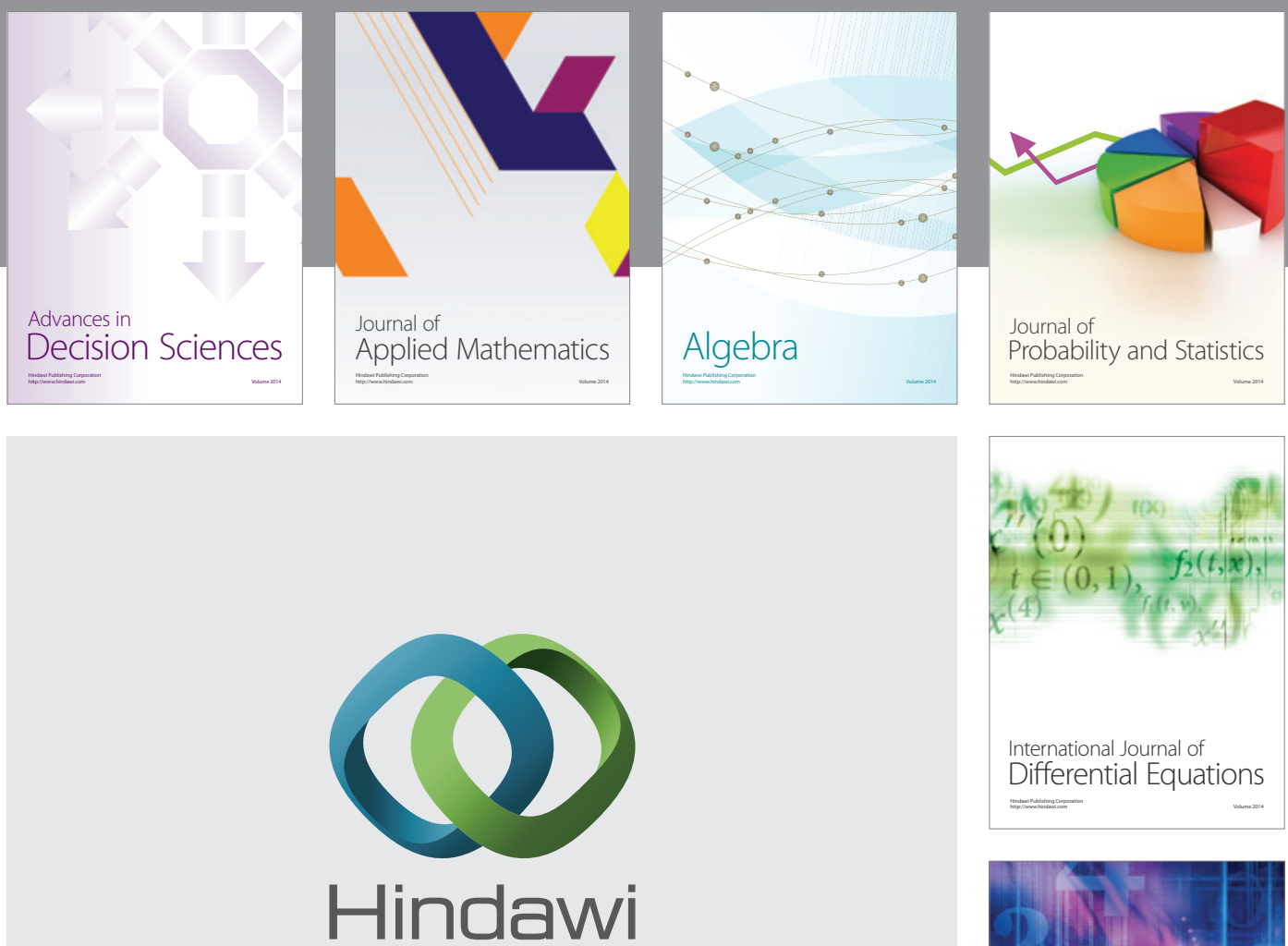

Submit your manuscripts at http://www.hindawi.com
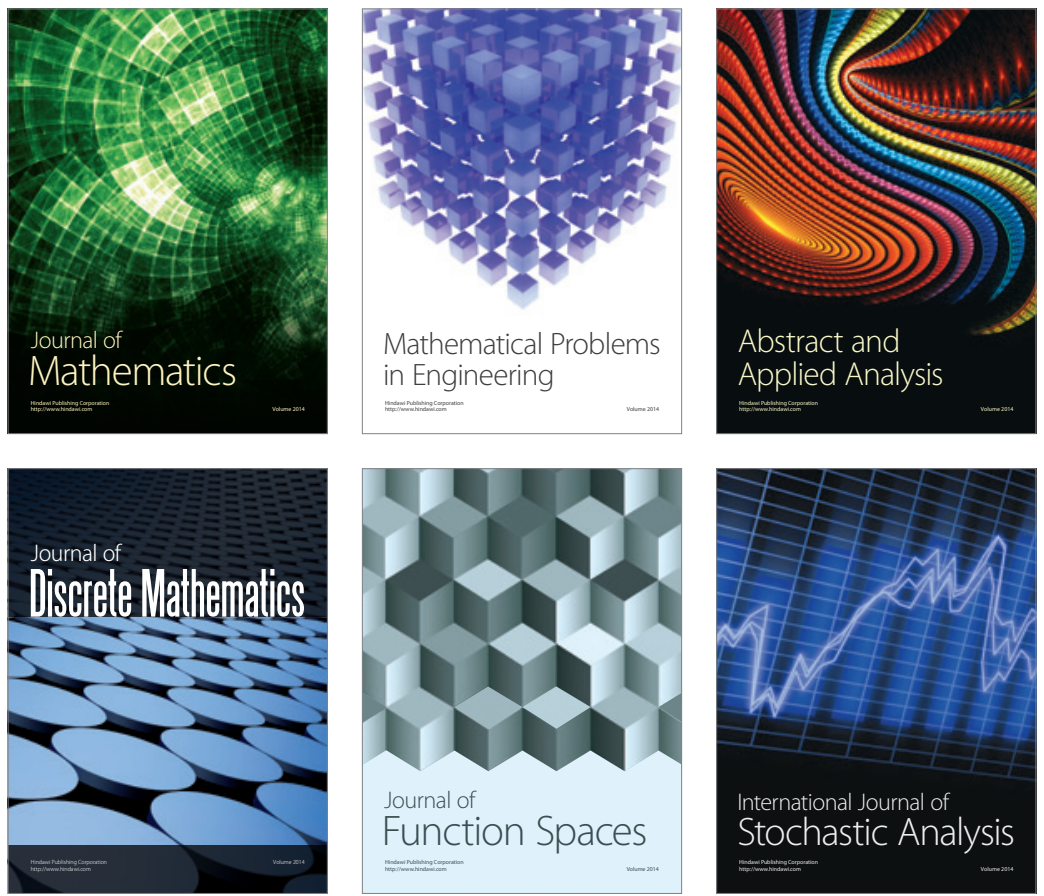

Journal of

Function Spaces

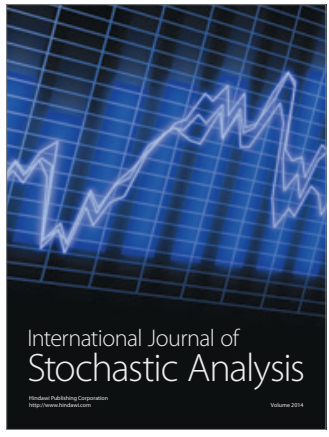

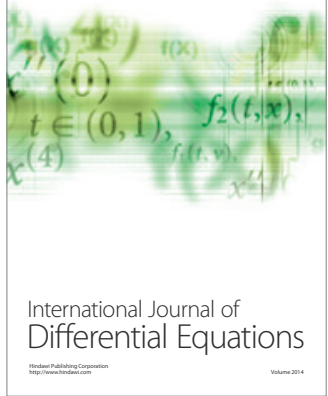
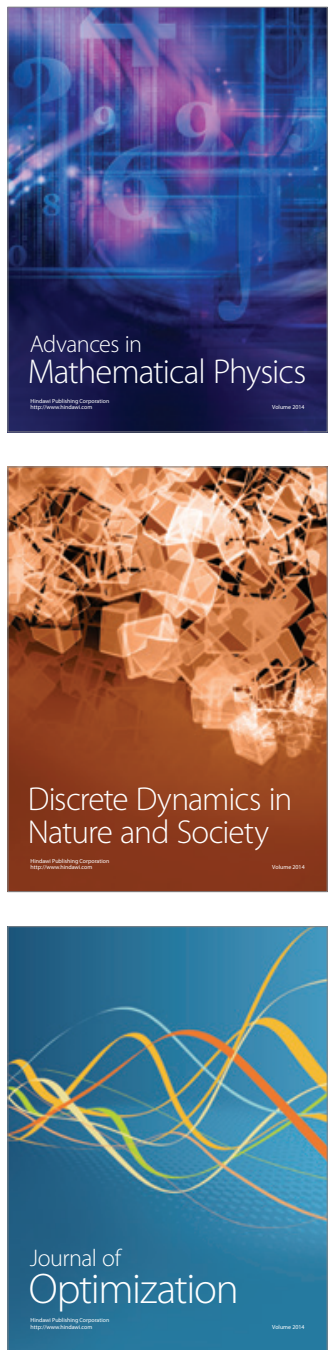